Revista electrónica de AnestesiaR

\author{
FORMACION MÉDICA
}

Febrero 2019

\title{
Retos en la UCI: El paciente muy anciano
}

Lagunes Herrero J.

\section{Consorcio Hospital General Universitario, Valencia.}

\begin{abstract}
Resumen
Debido a las limitaciones de recursos disponibles y la divergencia en el resultado final de los pacientes muy ancianos (edad > 80 años), estos son evaluados para valorar el pronóstico y el beneficio antes de proceder a su admisión en la UCI. Según los estudios de población es previsible un elevado aumento de pacientes de edad avanzada que requieran cuidados intensivos en un futuro próximo. Las decisiones de la admisión deberían orientarse según el pronóstico determinado por la fragilidad y la comorbilidad, pero con un enfoque más funcional. Siendo el índice de fragilidad un predictor independiente más significativo que la edad, la gravedad de la enfermedad e incluso la comorbilidad. La fragilidad es multidimensional, pero implica características cognitivas y de discapacidad que ayudan en la toma de decisiones para admitir a un paciente anciano en la UCI.

El estudio ICE-CUB 1 y el reciente estudio ICE-CUB 2 se mantienen los mismos resultados e implica que es necesario evaluar los posibles beneficios y daños de la admisión en la UCI de los pacientes muy ancianos. El presente artículo que solicito evalúen intenta orientar y ayudar para esa toma de decisiones en estos pacientes.
\end{abstract}

\section{Introducción}

Debido a las limitaciones de recursos disponibles y la divergencia en el resultado final de los pacientes muy ancianos (edad > 80 años), estos son evaluados para valorar el pronóstico y el beneficio antes de proceder a su admisión en la UCI. Según los estudios de población es previsible un elevado aumento de pacientes de edad avanzada que requieran cuidados intensivos en un futuro próximo. Las decisiones de la admisión deberían orientarse según el pronóstico determinado por la fragilidad y la comorbilidad, pero con un enfoque más funcional. Siendo el índice de fragilidad un predictor independiente más significativo que la edad, la gravedad de la enfermedad e incluso la comorbilidad. La fragilidad es multidimensional, pero implica características cognitivas y de discapacidad que ayudan en la toma de decisiones para admitir a un paciente anciano en la UCI.

El estudio ICE-CUB 1 y el reciente estudio ICE-CUB 2 se mantienen los mismos resultados e implica que es necesario evaluar los posibles beneficios y daños de la admisión en la UCI de los pacientes muy ancianos. El presente artículo que solicito evalúen intenta orientar y ayudar para esa toma de decisiones en estos pacientes.

El número de personas mayores de 80 años experimentará un incremento del $23 \%$ en el año 2029 y del $56 \%$ en el 2064, según análisis del INE (Instituto Nacional de Estadística) y Eurostat (1) ver gráfico 1 y tabla 1- para la población estimada años 2020-2080. Las unidades de cuidados intensivos (UCI) en la actualidad, a nivel mundial, tienen una demanda de ingresos de personas mayores de 80 años situada entre el $10-20 \%$ (2), por lo que es 
previsible que experimenten un incremento paralelo al aumento de la esperanza de vida. Unido a lo anterior, existe una incertidumbre en los resultados sobre la mortalidad y calidad de vida tras su ingreso en la UCI. Los estudios muestran divergencias, siendo incluso la mortalidad similar a los pacientes que no ingresan en la UCI (3, 4).

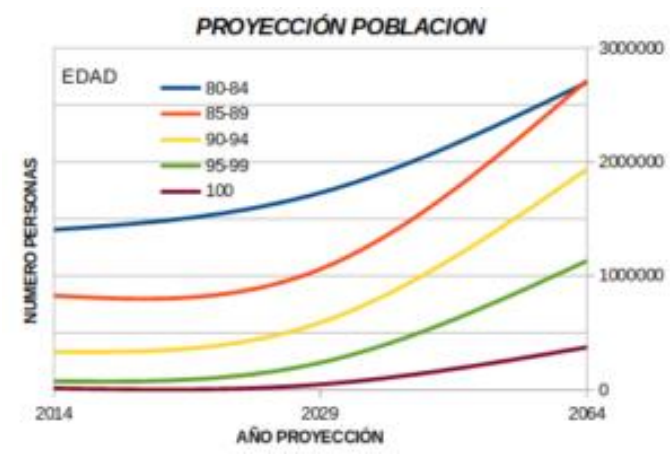

Imagen 1 (basado en los datos del INE)

\begin{tabular}{|c|c||}
\hline \hline \multicolumn{2}{|c||}{ Europa -28 > 80 años } \\
\hline $\mathbf{2 0 1 5}$ & $5,30 \%$ \\
\hline $\mathbf{2 0 3 0}$ & $7,10 \%$ \\
\hline $\mathbf{2 0 6 0}$ & $12,00 \%$ \\
\hline
\end{tabular}

Tabla 1: previsión de la población +80 años en la Eur-28.

La creciente demanda de cuidados intensivos en ancianos y los recursos limitados de la UCI, hacen previsible que la demanda y el gasto sanitario aumenten. Nuestros esfuerzos deben continuar para mejorar la atención a este grupo de pacientes y conseguir un control del gasto sostenible. A continuación se exponen los puntos clave del problema, con los datos disponibles, para evaluar a los pacientes que se beneficiarán de una terapia intensiva y la estrategia para mejorar los cuidados en su atención.

\section{Puntos Clave}

\section{Definir}

Lo primero sería definir quién es un paciente muy anciano (PMA), considerándose como referencia a los pacientes mayores 80 años, aunque podría ser más significativo definir aquel paciente con una esperanza de vida menor de 10 años, siempre dependiendo de la región o país de referencia. La decisión de admisión en la UCI de los PMA es difícil y no debe basarse solo en la edad como indica el estudio de Pierrick Le Borgne et al, realizado en pacientes $>90$ años (5), pues implica el riesgo de exceso o subutilización de las camas disponibles en una UCI y durante su estancia existe también el riesgo de infringir más daño que bien a estos pacientes. Tampoco debemos olvidar que existe una incertidumbre del resultado final respecto a la supervivencia y la calidad de vida (3).

\section{Identificar qué PMA se beneficiarán o no del ingreso en la UCI}

a.- Es necesario conocer los deseos del paciente y favorecer la comunicación con la familia, para explicarles el pronóstico y los riesgos asociados a su ingreso en la UCI. El estudio ICE-CUB 1 , un estudio observacional prospectivo multicéntrico de 2646 pacientes críticamente enfermos de 80 años o más, encontró que la admisión en la UCI no generó ningún beneficio de mortalidad a los 6 meses: $50.6 \%$ vs $50.7 \% \quad$ (3). Posteriormente la continuación del estudio francés ICECUB 2, un estudio clínico aleatorizado por grupos que estudió 3036 pacientes mayores críticamente enfermos -no oncológicos- (4), comparó la realización de un programa para promover la admisión sistemática en la UCI frente a seguir la práctica estándar o grupo control de atención habitual, así estos pacientes con frecuencia no recibieron cuidados de la UCI a pesar de cumplir con los criterios de admisión. En ambos grupos, la decisión final fue tomada a pie de cama por el médico responsable $\mathrm{y}$ un intensivista. Los resultados 
supusieron un aumento en el uso de la UCI, con una mortalidad hospitalaria del grupo intervención 451 (30\%) frente al grupo control $326(21 \%)$, y con una mortalidad a los 6 meses de 685 (45\%) y $588(39 \%)$ respectivamente, es decir no se redujo la mortalidad.

b.- Disponer de herramientas que nos ayuden a realizar una identificación fiable de los pacientes que se beneficiarán de un tratamiento intensivo, en referencia a las escalas de gravedad: APACHE, SAPS, SOFA, etc. En todos los sistemas de severidad actuales los PMA solo contribuyeron en una proporción menor dentro de la población de estudio (SAPS II edad media 57 años, APACHE III media edad 59 años), no hay variables relacionadas con la vejez, como la demencia y la discapacidad para las actividades de la vida diaria, y por último la precisión de las escalas disminuye a medida que los pacientes son de mayor edad (6).

La escala más fiable debería evaluar la fragilidad de los PMA. Destacar el artículo de actualidad de ME. GordoFlores y D. Paz-Martín: “¿Qué deberías saber de tu paciente anciano vulnerable?" (7), ofrecen una visión actual del problema que supone el paciente anciano, la fragilidad, la anestesia y los cuidados postoperatorios. Por otro lado, el interesante estudio de cohorte prospectivo transnacional (8) de octubre de 2016 a mayo de 2017, con 30 días de seguimiento realizado por la Sociedad Europea de Cuidados Intensivos (ESICM), con la participación de 311 UCIs de 21 países donde se incluyeron los primeros 20 pacientes $\geq 80$ años que ingresaron en la UCI. Se registraron el índice de fragilidad (Clinical Frailty Scale-CFS-) (9), la escala SOFA (Sequential Organ Failure Assessment) y los procedimientos terapéuticos de 5021 pacientes con una edad media de
84 años. Una herramienta simple como CFS diferenció a los pacientes con una puntuación entre 5-9 como muy frágiles y con una mortalidad del $60 \%$, lo que proporciona ayuda en la evaluación de los PMA y es un factor significativo en la supervivencia a los 30 días.

Recientemente publicado por el Servicio de Intensivos del H. U. del Henares (10), sobre pacientes mayores de 74 años (mediana de 80 años), los factores asociados de forma independiente con la mortalidad o mala situación funcional al alta del hospital fueron la situación funcional previa al ingreso y a la gravedad al ingreso evaluada por SAPS 3.

c.- Ingreso de prueba: Cuando el beneficio del ingreso en la UCI es incierto o dudoso, el ingreso de prueba puede ser una alternativa. Deberá establecerse un periodo corto de $24-48 \mathrm{~h}$ de seguimiento y reevaluación para continuar la terapia intensiva o transferir al paciente a otra unidad con un tratamiento conservador o en su caso paliativo.

\section{Mortalidad elevada}

La mortalidad en la UCI es alta en los PMA a pesar del tratamiento intensivo, la mortalidad intrahospitalaria temprana se sitúa entre el 25 y $45 \%$ y la mortalidad a 1 año puede alcanzar el $70 \%$ (5). Además, debemos saber que la recuperación del estado funcional es menor. En un estudio prospectivo de cohortes multicéntrico en 22 UCIs de Canadá (11) se siguió la evolución durante un año de 610 PMA, de media los pacientes pasaron algo menos de 7 días en la UCI y 3 semanas en el hospital. Aproximadamente el $20 \%$ murió en la propia UCI, un $12-15 \%$ de pacientes dados de alta de la UCI murió en el hospital y al año el 50\% había muerto. Pero de los pacientes supervivientes solo el $26 \%$ 
habían recuperado su nivel físico basal. Véase otro ejemplo del grafico 2: mortalidad por sepsis grave con ingreso en UCI y en el hospital según tramos por edad, estudio de Haas L.E.M et al. (12)

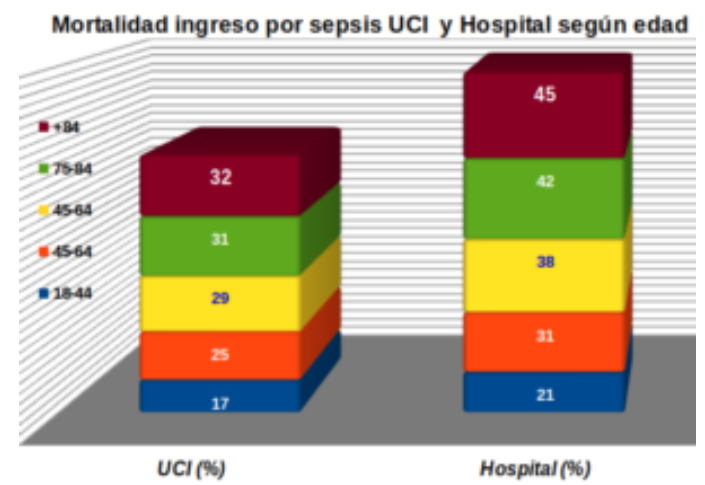

Imagen 2. Gráfico propio tomados los datos de Dimopoulos 2013 (referenciado en 10)

También es importante diferenciar el ingreso programado del urgente. El trabajo multicéntrico de la ESICM -311 ICUs de 21 países, en el que participaron 16 UCIS españolas (8), nos proporciona los siguientes datos:

\begin{tabular}{|l|l|l|}
\hline Variable/ Ingreso & Electivo & Urgente \\
\hline Supervivencia UCI (\%) & 96,9 & 73,7 \\
\hline Supervivencia 30 días (\%) & 93,2 & 62 \\
\hline Estancia UCI (dias) & 1,2 & 2,8 \\
\hline Paciente fragiles (CFS $>5$ ) & 28,6 & 46,1 \\
\hline
\end{tabular}

Tabla 2

Es decir, una mortalidad del $23,2 \%$ para el ingreso urgente frente al $3,1 \%$ del programado. Siendo la diferencia de mortalidad mayor a los 30 días con un $31 \%$ y con un cociente de riesgo instantáneo (HR) para el ingreso urgente de 6,72 .

Además del ingreso urgente versus programado, debemos diferenciar el ingreso médico del quirúrgico. Los estudios demuestran que los pacientes quirúrgicos electivos o programados tienen mayor probabilidad de sobrevivir frente a los pacientes con patologías médicas, con un cociente de riesgo del 4,37 para el paciente médico (11). Respecto a la mortalidad, entre pacientes urgentes médicos $y$ quirúrgicos, la mortalidad en la UCI fue menor en un $8 \%$ del quirúrgico, y la mortalidad hospitalaria del $41.5 \%$ y $31.6 \%$ del paciente médico respecto del quirúrgico (13). Es decir, los pacientes médicos tienen peor pronóstico y mayor mortalidad tanto en la UCI como en el hospital respecto a los pacientes quirúrgicos.

\section{Puntos esenciales para valorar la admisión a en UCI}

Los datos disponibles en la actualidad solo están basados en estudios observacionales y de cohorte, es notoria la ausencia de trabajos randomizados y aleatorios (14), salvo el estudio realizado por Guidet et al (4) que proporciona los primeros datos aleatorizados sobre los efectos de la atención en la UCI de pacientes ancianos críticamente enfermos. Por lo que quedan muchas preguntas en el aire, respecto a la decisión de admitir un PMA en la UCI: ¿puede mejorarse el tratamiento de estos pacientes en las plantas del hospital?, ¿sería bueno la creación de UCIs geriátricas y con un nivel de soporte de órganos menor?, ¿debería integrarse un geriatra $o$ internista en los cuidados?, ¿debería existir mayor comunicación entre los profesionales de las distintas especialidades y los intensivistas para adecuar un tratamiento menos invasivo? (15).

Estas y otras muchas preguntas son y serán tema de debate y discusión, pero debemos avanzar en el conocimiento y los retos de nuestra práctica clínica habitual. Por lo que a continuación se exponen los puntos que pueden ayudar en la decisión de admitir a un PMA en la UCI: 
1.- Una buena comunicación con el paciente y su familia. Muchos pacientes ancianos rechazan un tratamiento más agresivo y suelen preferir uno que les proporcione mayor confort y la compañía de su familia. Esto podría limitar el uso de tratamientos fútiles, costosos y prolongados para el mantenimiento de la vida.

2.- Identificar a los PMA que podrían beneficiarse del ingreso en la UCI, independientemente de la edad, atendiendo al índice de fragilidad, la discapacidad, la patología crónica de base y diagnóstico de ingreso actual.

* Escala clínica de fragilidad: con una puntuación de la escala CFS $<3$ paciente no frágil, a $>5$ puntos del paciente frágil.

* Clase funcional: Barthel o de discapacidad de Maryland $>90$ puntos (buena) a menor número más discapacidad, y el índice de Katz A y B (máxima dependencia letra $\mathrm{G}$ ).

* Patología crónica y terapia actual: oxigenoterapia domiciliaria, transfusión de hemoderivados frecuentes, hemodiálisis, etc.

* Pronóstico (de mejor a peor): quirúrgico electivo, quirúrgico urgente y por último médico.

* Soporte de órganos necesario: sabiendo que según el estudio de Flaatten H (8) de la ESICM, los procedimientos más frecuentes fueron el uso de fármacos vasoactivos en el $52,2 \%$, la ventilación invasiva en el $50,7 \%$, la VNI se utilizó en el $23 \%$, mientras que la terapia de reemplazamiento renal se utilizó solo en el $9,2 \%$ de todos los ingresos.

3.- Valorar el ingreso de prueba en los casos que existan dudas respecto al pronóstico: consensuar 24-48 h de admisión y los niveles del tratamiento a establecer. Posteriormente reevaluar.

4.- Si se decide que el paciente podría beneficiarse de un ingreso de prueba pero no existe cama disponible en la UCI, valorar si es posible el apoyo en planta mediante el equipo de Servicio Ampliado de Cuidados Críticos (SACC), o si sería preferible desplazar al paciente a otro hospital con cama de UCI. Si no es posible optimizarlo, debería promoverse el traslado a otro hospital consensuando el nivel de atención previsto.

5.- Establecer un programa de actuación del PMA una vez admitido, valorando el tratamiento y nivel máximo de soporte de órganos, sin caer en alargar una situación agónica. Reevaluación cada 48-72 h para valorar continuar con tratamientos intensivos o derivar al paciente, si esto es posible, a una unidad o planta de hospitalización y continuar un tratamiento conservador o paliativo en su caso.

6.- Ante el fracaso en el tratamiento intensivo, proporcionar una adecuada atención al final de la vida al paciente y favorecer la intimidad familiar.

7.- Seguimiento del paciente una vez dado de alta, es importante obtener datos de nuestra actuación, de los resultados inmediatos y también a largo plazo: mortalidad hospitalaria, alta hospitalaria y estado funcional.

\section{Bibliografía}

- INE. Last access Julio de 2018. Nota de prensa.28/10/2014.

http://www.ine.es/prensa/np870.pdf

EUROSTAT Population structure and ageing http:/ec.europa.eu/eurostat/statistics-

explained/index.php?title=Population_structure and ageing\#The share_of_elderly_people_co ntinues to increase 
- Ihra GC, Lehberger J, Hochrieser H, Bauer P, Schmutz R, Metnitz B et al. Development of demographics and outcome of very old critically ill patients admitted to intensive care units. Intensive Care Med 2012; 38:620-626. (PubMed)

- Boumendil A, Angus DC, Guitonneau AL, Menn AM, Ginsburg C, Takun K et al ICECUB study group. Variability of intensive care admission decisions for the very elderly. PLoS One. 2012;7(4): e34387. (PubMed) ( (PDF)

- Guidet B, Leblanc G, Simon T, Woimant M, Quenot JP, Ganansia $O$ et al. Effect of Systematic Intensive Care Unit Triage on Longterm Mortality Among Critically Ill Elderly Patients in France: A Randomized Clinical Trial. JAMA. 2017;318(15):1450-1459. (PubMed) ( $\underline{\text { HTML) }}$

- Le Borgne P, Maestraggi Q, Couraud S, Lefebvre F, Herbrecht JE, Boivin A et al. Critically ill elderly patients $(\geq 90$ years): Clinical characteristics, outcome and financial implications. Lazzeri C, ed. PLoS ONE. 2018;13(6): e0198360. ( PubMed)

- Flaatten H, de Lange W.D. Artigas A, Bin D, Moreno R, Christensen $\mathrm{S}$ et al. The status of intensive care medicine research and a future agenda for very old patients in the ICU. Intensive Care Medicine. 2017; 43 (9):13191328. ( $\underline{\text { PubMed }})$

- Gordo-Flores M.E, Paz-Martín D. Fragilidad en Anestesia y Cirugía. ¿Qué deberías saber de tu paciente anciano vulnerable? https://anestesiar.org/2017/fragilidad-enanestesia-y-cirugia-que-deberias-saber-de-tupaciente-anciano-

vulnerable/?utm_source=blogsterapp\&utm_med ium $=$ twitter $(\underline{\text { HTML }})$

- Flaatten H, De Lange DW, Morandi A, Andersen FH, Artigas A, Bertolini G et al. The impact of frailty on ICU and 30-day mortality and the level of care in very elderly patients $(\geq$ 80 years). Intensive Care Med. 2017; 43: 18201828. ( PubMed)

- Rockwood K, Song X, MacKnight C, Bergman H, Hogan D.B., McDowell I et al. A global clinical measure of fitness and frailty in elderly people. CMAJ. 2005; 173 (5): 489-495. (PubMed) ( $\underline{\text { HTML) }}$ ( $\underline{\text { PDF }})$
- Gordo F, Castro C, Inés Torrejón I, Bartolomé $\mathrm{S}$, Coca $\mathrm{F}$ and Abella A. Situación funcional como factor independiente asociado con el mal pronóstico de pacientes ancianos ingresados en Cuidados Intensivos. Revista Española de Geriatría y Gerontología. 2018, 53 (4): 213-216. (HTML)

- Heyland DK, Garland A, Bagshaw SM, Cook D, Rockwood K, Stelfox HT et al. Recovery after critical illness in patients aged 80 years or older: a multi-center prospective observational cohort study. Intensive Care Med. 2015; 41:1911-1920. ( PubMed)

- Haas L.E.M., van Dillen L. S, de Lange, van Dijk D.W. D, Hamaker M.E. Outcome of very old patients admitted to the ICU for sepsis: A systematic review. European Geriatric Medicine. 2017; 8(5-6): 446-453.

- Ball IM, Bagshaw SM, Burns KE, Cook DJ, Day AG, Dodek PM et al. Outcomes of elderly critically ill medical and surgical patients: a multicentre cohort study. Can J Anesth/J Can Anesth 2017; 64: 260-269. (PubMed) https://www.ncbi.nlm.nih.gov/pubmed/2802867 $\underline{3}$

- Angus DC. Admitting Elderly Patients to the Intensive Care Unit-Is it the Right Decision? JAMA. 2017;318(15):1443-1444. (ubMed)

- Zeng A, Song X, Dong J, Mitnitski A, Liu J, Guo $Z$ et al. Mortality in Relation to Frailty in Patients Admitted to a Specialized Geriatric Intensive Care Unit. The Journals of Gerontology Series A: Biological Sciences and Medical Sciences. 2015;70(12):1586-1594. doi:10.1093/gerona/glv084. (uㅏㄹed)

Correspondencia al autor

José LLagunes Herrero

josellagunes@gmail.com

Servicio de Anestesia, Cuidados Críticos y Terapia del Dolor

Consorcio Hospital General Universitario, Valencia

Aceptado para blog en septiembre de 2018. 